\title{
Using the tetravalent meningococcal polysaccharide-protein conjugate vaccine in the prevention of meningococcal disease
}

\author{
Sanford R Kimmel \\ University of Toledo College \\ of Medicine, Department of Family \\ Medicine, Toledo, $\mathrm{OH}$, USA
}

\begin{abstract}
Invasive meningococcal disease occurs worldwide causing an estimated 50,000-135,000 deaths each year in addition to significant sequelae. In developed countries the disease is usually sporadic but outbreaks and epidemics, usually due to serogroups B and C, have occurred. In the US, an increasing number of cases are due to serogroup Y. In developing nations, epidemics due to serogroups A and more recently W-135, are common. The tetravalent meningococcal conjugate vaccine to serogroups A, C, Y, and W-135 (MCV4) has been demonstrated to be highly immunogenic and promote immune memory. This article will describe the rationale for the vaccine and its potential role to significantly decrease mortality and morbidity of meningococcal disease in those areas and populations at greatest risk from these serogroups.
\end{abstract}

Keywords: meningococcal disease, tetravalent meningococcal conjugate vaccine, serogroup

\section{Epidemiology and pathogenesis}

Neisseria meningitidis is an aerobic, gram negative diplococcus that colonizes the nasopharynx of humans and is transmitted by respiratory tract droplets (Gardner 2006). Almost all human disease is caused by 5 of the 13 serogroups: A, B, C, Y, and W-135. Worldwide, approximately 500,000 cases of invasive meningococcal disease (IMD) occur annually resulting in an estimated 50,000-135,000 deaths per year (Pollard 2004; Borrow and Miller 2006). The incidence of the disease in developed countries varies from 0.5 to 1.1 per 100,000 people per year in the US (Gardner 2006) to 3.6 cases per 100,000 in England and Wales (Pollard 2004). In 2006, the estimated incidence of probable and confirmed IMD varied from 0.30 and 0.35 per 100,000 people in Italy and Hungary, respectively, to 4.96 and 8.90 per 100,000 in Ireland and Malta, respectively (EU-IBIS 2006). In the US, over $98 \%$ of cases of IMD are sporadic (Bilukha and Rosenstein 2005) but the disease is epidemic in many parts of the world.

The influence of serogroups in causing meningococcal disease varies according to year, geography, and, in some cases, ethnic and socioeconomic factors. Serogroups $\mathrm{B}$ and $\mathrm{C}$ cause most cases of sporadic and rare epidemic disease in developed countries. Serogroups B, C, W-135, and Y comprised 61\%, 32.2\%, 3.2\%, and 2.9\% of meningococcal isolates, respectively, from IMD patients in the Czech Republic, Greece, and Norway (Yazdankhah et al 2004). Among IMD isolates, serogroup B predominated in Greece and Norway while serogroup C predominated in in the Czech Republic (Yazdankhah et al 2004). Serogroup A and recently W-135 cause most cases of epidemic disease in sub-Saharan Africa (almost 1000 cases per 100,000 population) (Pollard 2004). Serogroup W-135 meningococcal disease has been reported in pilgrims returning from the Hajj in Saudi Arabia and in their contacts (MMWR 2001b; Hahne 2002). Serogroup Y accounted for only $2 \%$ of meningococcal disease in the US from 1988 to 1991 but accounted for $40 \%$ of cases during 2006, while serogroups B and C 
accounted for $28 \%$ and $27 \%$ during that year (CDC 2007). In New Zealand, an epidemic of predominantly serogroup B meningococcal disease has recorded disease rates as high as approximately 500 per 100,000 in Pacific children less then 1 year of age compared with less then 50 per 100,000 children of European descent (O’Hallahan et al 2004).

Invasive meningococcal disease usually occurs in persons who are seronegative and subsequently acquire $N$. meningitidis from crowding such as among military recruits and freshmen college students living in residence halls (Bruce et al 2001). The nasopharyngeal carriage rate of $N$. meningitidis has been shown to increase rapidly for first year university students living in residence halls (Neal et al 2000). Exposure to tobacco smoke and alcohol-related behaviors are other potential risk factors for meningococcal disease in this population (Harrison et al 1999).

Once $N$. meningitidis attaches to the nasopharyngeal mucosal cells, replicates, and establishes a carrier state, multiple factors determine the occurrence of IMD including the virulence of the organism and factors affecting host susceptibility (Granoff 2004). The spleen is responsible for clearing meningococci from the blood and anatomic splenectomy has resulted in overwhelming meningococcal sepsis (Condon et al 1994). Patients with functional hypoor asplenia are also at increased risk for disease (Granoff 2004). Deficiencies of terminal complement component, properdin, factor $\mathrm{D}$, and mannan-binding lectin (MBL) have been associated with increased susceptibility to meningococcal infection (Pollard and Frasch 2001). The absence of serum bactericidal antibodies (SBAs) to human complement (hSBAs) to serogroup C meningococci, as demonstrated by a titer of less then 1:4, was associated with an increased risk of meningococcal disease in military recruits (Goldschneider et al 1969a). It was later demonstrated that SBAs to rabbit complement (rSBAs) with a titer of less then 1:8 also predict susceptibility to meningococcal disease (Borrow et al 2001). Conversely, a hSBA titer to serogroup $\mathrm{C}$ of $\geq 4$ is considered protective while a rSBA titer of $\geq 8$ is correlated with at least short-term protection (Borrow et al 2005).

\section{Burden of illness}

Vieusseux in Switzerland first described epidemic meningococcal disease in 1805, but Weichselbaum first identified the bacterium in 1887 (Barquet et al 1999; Rosenstein et al 2001). Meningitis occurs in about $50 \%$ of patients, and in older children and adults is accompanied by sudden headache, fever, stiff neck, and sometimes nausea, vomiting, and photophobia (Rosenstein et al 2001). In infants and very young children, fever, poor feeding, and lethargy may be seen initially while nuchal rigidity presents later in the course of the illness (Granoff 2004). A rash is often present but may vary from maculopapular to the classic petechial or purpuric rash. Meningococcal sepsis or meningococcemia occurs in $5 \%-20 \%$ of IMD (Rosenstein et al 2001). It is characterized by sudden fever, petechial or purpuric rash, hypotension progressing to shock, acute adrenal hemorrhage (Waterhouse-Friederichsen syndrome), and multiorgan failure (Rosenstein, Perkins et al 2001). Other forms of IMD include pneumonia, arthritis, pericarditis, and cellulitis (van Deuren et al 2000).

The overall case-fatality rate for IMD in the US is approximately $9 \%-12 \%$, even with appropriate antibiotic treatment, and that for meningococcemia is as high as $40 \%$ (Rosenstein et al 2001). However, the fatality rate can vary according to the affected population and the meningococcal serogroup. In New Zealand, the average case-fatality rate for serogroup B IMD is 4.3\% (O'Hallahan et al 2004). In a study of 295 cases of IMD in Maryland, USA from 1992 to 1999 , persons ages $15-24$ years old accounted for $24 \%$ of IMD cases and had a fatality rate of $23 \%$ compared with $4.6 \%$ of those younger than 15 years and $17 \%$ of those older than 24 years (Harrison et al 2001). Of the 14 fatalities for which serogroup was known, serogroups $\mathrm{C}$ and $\mathrm{Y}$ accounted for $57 \%$ and $29 \%$, respectively, of fatalities in this age group (Harrison et al 2001). Consequently, more then $85 \%$ of these deaths were potentially preventable by the tetravalent (Groups A, C, Y, and W-135) meningococcal vaccines.

Invasive meningococcal disease may also cause sequelae such as hearing loss, neurologic deficits, or limb loss in up to $11 \%-19 \%$ of cases (Rosenstein et al 2001). A review of IMD in Barcelona from 1987 to 1992 found a sequelae rate of $8.2 \%$ for meningitis, $3.5 \%$ for meningococcemia with meningitis, and $1.5 \%$ for meningococcemia with an overall rate of $4 \%$ (Barquet et al 1999). All patients who had neurologic sequelae had meningitis while meningococcemia was associated with sequelae such as limb amputation (Barquet et al 1999).

\section{Outbreaks of meningococcal disease}

Household contacts of patients with IMD have a 500-800 times greater risk of meningococcal disease than the general rate and should be given chemoprophylaxis (Bilukha and Rosenstein 2005). They are candidates for immunization with meningococcal vaccine, but this does not substitute for chemoprophylaxis because of the time delay in developing immunity. Chemoprophylaxis should also be given to close 
contacts including child-care center contacts, anyone directly exposed to the patient's oral or respiratory secretions, and passengers who sat on either side of the index-patient on a flight lasting $>/=8$ hours (MMWR 2001a; Bilukha and Rosenstein 2005). Because systemic therapy other than ceftriaxone or other third-generation cephalosporin might not eradicate $N$. meningitidis from the nasopharynx, patients treated for IMD with other agents such as penicillin should also receive chemoprophylaxis (Abramson and Spika 1985; Bilukha and Rosenstein 2005). Chemoprophylaxis is ideally given within 24 hours of identification in the index patient and is of little value $>14$ days after disease onset (Bilukha and Rosenstein 2005). Table 1 lists currently recommended antibiotic prophylaxis for meningococcal disease. Fluoroquinolone-resistant $N$. meningitidis has been reported in Argentina, Australia, China, France, India, Spain, and most recently in the northern US (MMWR 2008). One study found azithromycin comparable in efficacy to rifampin in eradicating nasopharyngeal coverage of $N$. meningitis (Girgis 1998). When fluoroquinolone-resistant meningococcal disease is identified, interim $\mathrm{CDC}$ recommendations are to give a single dose of $500 \mathrm{mg}$ azithromycin for exposed persons $\geq 15$ years old or $10 \mathrm{mg} / \mathrm{kg}$ body weight for those under 15 years old (MMWR 2008).

The United States Centers for Disease Control and Prevention (CDC) define outbreaks as three or more confirmed or probable cases of meningococcal disease in $\leq 3$ months among persons residing in the same area who are not close contacts of each other with a primary disease attack rate of $\geq 10$ cases/100,000 persons (Bilukha and Rosenstein 2005). In the circumstance of a confirmed outbreak, public health personnel should consider vaccinating the population at risk. Mass chemoprophylaxis is not recommended except for outbreaks involving populations in a limited setting such as school or for a serogroup B outbreak in those countries where a vaccine is not available (Bilukha and Rosenstein 2005).

\section{Meningococcal polysaccharide vaccines}

Goldschneider and colleagues demonstrated that transplacental passage of antibodies to meningococci occurred resulting in passively acquired immunity to infection (Goldschneider et al 1969b). In addition they found that military recruits developed bactericidal antibodies to their own meningococcal isolates as a response to their carrier state (Goldschneider et al 1969b). Animal and human testing subsequently demonstrated that serogroups A and C polysaccharides stimulated immunogenicity (Gotschlich et al 1969). This led to the development of polysaccharide vaccines that were effective in preventing group $\mathrm{C}$ disease in US military recruits and controlling group A epidemics in Africa (Jodar et al 2002). The tetravalent polysaccharide vaccine to serogroups A, C, Y, and W-135 (MPSV4, Menomune ${ }^{\circledR}-\mathrm{A}, \mathrm{C}, \mathrm{Y}, \mathrm{W}-135$, Sanofi Pasteur, Inc., Swiftwater, Pennsylvania, US) was 85\% efficacious in preventing group $\mathrm{C}$ meningococcal disease during an outbreak in Texas, USA (Rosenstein et al 1998).

The MPSV4 vaccine is T cell-independent and the serogroup $\mathrm{C}$ component is poorly immunogenic in children less then 18-24 months old while the serogroup A polysaccharide does not induce an immune response comparable to adults until ages 4-5 years (MMWR 2000). In children less then 5 years, antibody levels to group $\mathrm{A}$ and $\mathrm{C}$ polysaccharides decrease significantly within 3 years after a single vaccine dose (MMWR 2000). The vaccine also does not stimulate immune memory, and revaccination with serogroup C capsular polysaccharide vaccines often results in a hypo-responsive

Table I Chemoprophylactic agents for meningococcal disease

\begin{tabular}{|c|c|c|c|}
\hline Drug & Age group & Dosage and route & Duration \\
\hline Rifampin $^{\mathrm{a}}$ & Children $<$ I month & $5 \mathrm{mg} / \mathrm{kg}$ orally every $12 \mathrm{~h}$ & 2 days \\
\hline \multirow[t]{2}{*}{ (Rifadin) } & Children $>$ I month & $10 \mathrm{mg} / \mathrm{kg}$ orally every $12 \mathrm{~h}$ & 2 days \\
\hline & Adults & $600 \mathrm{mg}$ orally every $12 \mathrm{~h}$ & 2 days \\
\hline Ciprofloxacin $^{\mathrm{b}}\left(\right.$ Cipro $\left.^{\circledast}\right)$ & Adults & $500 \mathrm{mg}$ orally & Single dose \\
\hline \multirow[t]{2}{*}{ Ceftriaxone $\left(\right.$ Rocephin $\left.{ }^{\circledR}\right)$} & Children $<15$ years & $125 \mathrm{mg}$ intramuscularly & Single dose \\
\hline & Persons $\geq 15$ years & $250 \mathrm{mg}$ intramuscularly & Single dose \\
\hline \multirow[t]{2}{*}{ Azithromycin ${ }^{c}$} & Children $<15$ years & 10 mg/kg body weight & Single dose \\
\hline & Persons $\geq 15$ years & 500 mg & Single dose \\
\hline
\end{tabular}

aRifampin is not recommended for pregnant women because it is teratogenic in laboratory animals. It may decrease the effectiveness of other medications, including oral contraceptives, and alternative contraceptive methods are recommended during its use.

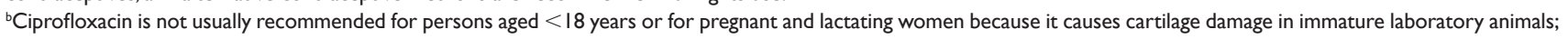
however, it can be used for chemoprophylaxis of children when no acceptable alternative therapy is available.

${ }^{\mathrm{c} A z i t h r o m y c i n}$ is recommended as an alternative agent for chemoprophylaxis in certain areas of the US (North Dakota and Minnesota) where fluoroquinolone-resistant meningococcal disease has been identified.

Derived from Bilukha and Rosenstein (2005) and MMWR (2008). 
antibody response to group $\mathrm{C}$ polysaccharide in children and adults (Granoff et al 1998; MacDonald et al 1998; Danzig 2004). It has been posited that repeated stimulation of B cells with the T-cell independent plain polysaccharide vaccine results in terminal differentiation of $B$ cells to produce plasma cells and depletes the pool of specific B cells (Granoff and Pollard 2007).

The serogroup B meningococcal polysaccharide is poorly immunogenic and the resulting IgG antibodies have poor complement bactericidal activity. In addition, the serogroup B capsular polysaccharide is structurally similar to a nerve cell molecule present in developing neural tissue and small amounts of adult tissue (Danzig 2004). Vaccines for serogroup B that utilize the outer membrane vesicle (OMV) of $N$. meningitidis are under trial in a number of countries (Borrow et al 2005). Their use is beyond the scope of this article.

\section{The United Kingdom (UK) experience with conjugate meningococcal $C$ vaccine}

Polysaccharides conjugated to carrier proteins induce T-cell dependent immunity (Granoff 2004). In November, 1999, the UK initiated a program to vaccinate all children with conjugate meningococcal $\mathrm{C}$ vaccine. Two vaccine brands used CRM197 as the carrier protein (Wyeth and Novartis, Canada, QC) and one used tetanus toxoid (Baxter Bioscience, IL, USA) (Borrow and Miller 2006). The vaccine was given to infants at 2,3 , and 4 months of age, with a single dose catch-up to all children ages 1-18 years old and two doses for infants 5-11 months old (Borrow and Miller 2006). These vaccines were demonstrated to be immunogenic and efficacious in infants, children, and adolescents. The number of cases of serogroup C IMD in adolescents 15-17 years old decreased by $76 \%$ from Jan 1 to Sept 30, 2000 compared with the corresponding period in 1999 and vaccine efficacy was estimated at $97 \%$ in teenagers (Ramsay et al 2001). Cases of serogroup C IMD in toddlers decreased by $34 \%$ during this period, but vaccine efficacy was estimated at 92\% (Ramsay et al 2001). More than 1 year later, vaccine effectiveness decreased significantly in infants 2-4 months at initial immunization while effectiveness in older children and especially adolescents persisted (Trotter et al 2004).

The nasopharyngeal carriage of meningococcal serogroup $\mathrm{C}$ bacteria in students aged 15-17 years was found to decrease by $66 \% 1$ year later (Maiden and Stuart 2002). The attack rate of serogroup C IMD subsequently decreased by $67 \%$ overall in un-immunized children and adolescents from July 2001 to June 2002 compared with July 1998 to June 1999 (Ramsay et al 2003). Consequently, there is evidence of herd immunity in unvaccinated members of the population or those in whom antibody levels have waned if a high enough proportion of the population is vaccinated.

\section{Safety and efficacy of the tetravalent meningococcal conjugate vaccine}

Three separate groups of 30 healthy US adults ages 18-55 years were given a single dose of tetravalent meningococcal conjugate vaccine containing either 1,4 , or $10 \mu \mathrm{g}$ of each capsular polysaccharide conjugated to diphtheria toxoid. Twenty-eight days after immunization, the percentage of recipients with SBA titers of $\geq 8$ was $100 \%, 89 \%-100 \%$, $88 \%$, and $91 \%$ for serogroups A, C, Y, and $\mathrm{W}-135$, respectively (Campbell et al 2002). Mild to moderate local reactions such as injection site erythema and swelling occurred in over $50 \%-90 \%$ of subjects and in each dose group, $41 \%-50 \%$ reported at least one systemic reaction such as headache and malaise. One subject experienced severe pain, chills, and malaise with local tenderness and vomiting that resolved within 2 days (Campbell et al 2002). Another study randomized over 800 adolescents (ages 11-18 years) to MPSV4 or the meningococcal conjugate vaccine containing $4 \mu \mathrm{g}$ of capsular polysaccharide each from serogroups A, C, Y, $\mathrm{W}-135$ (MCV4). Both vaccines were highly immunogenic to all four serogroups, although the MCV4 recipients reported more local reactions than the MPSV4 group (69\% vs 30\%) (Keyserling et al 2005). Follow-up at 3 years demonstrated persistence of SBA and booster responses to MCV4 consistent with immune memory that was not present in the MPSV4 group (Keyserling et al 2005). In January 2005, the US Food and Drug Administration (FDA) licensed Meningococcal Polysaccharide (serogroups A, C, Y, and W-135) Diphtheria Toxoid Conjugate Vaccine (MCV4, Menactra ${ }^{\circledR}$, Sanofi Pasteur, Inc., Swiftwater, PA, US) for the immunization of adolescents and adults aged 11-55 years age (Bilukha and Rosenstein 2005).

A US study compared safety and immunogenicity of MCV4 with MPSV4 in 2- to 10-year-old children. The percentage of children with $\mathrm{SBA}$ titers $\geq 1 / 128$ ranged from $81 \%-97 \%$ across the 4 serogroups in the MCV4 group versus 73\%-93\% for the MPSV4 group (Pichichero et al 2005). Mild to moderate local and systemic reactions of short duration were common and comparable in both groups (Pichichero et al 2005). A subgroup of children vaccinated with MCV4 were given a challenge dose of MPSV4 23-36 months later. They demonstrated an increase in IgG 
antibody and antibody avidity consistent with the induction of immune memory (Pichichero et al 2006). A UK study gave MCV4 to children 2 to $<5$ years old who had previously received the monovalent meningococcal $\mathrm{C}$ conjugate vaccine. All 44 children developed SBA titers $\geq 8$ to all four serogroups with a booster response observed to serogroup C (El Bashir et al 2006). In October, 2007 the FDA subsequently licensed MCV4 for use in children aged 2-10 years (MMWR 2007).

Another study compared vaccine doses of 1,4 , and $10 \mu \mathrm{g}$ for each of the four polysaccharide serogroups conjugated to diphtheria toxoid that were given to infants at 2,4 , and 6 months of age. Presumed protective SBA levels $>/=1: 8$ varied from $54 \%$ to $92 \% 1$ month after the primary series depending upon vaccine serogroup and polysaccharide dose. A polysaccharide content of $10 \mu \mathrm{g}$ was associated with more frequent local reactions but not with a corresponding increase in immunogenicity than lower doses of each serogroup (Rennels et al 2004). Consequently, there is no advantage to having a higher dose of the polysaccharide serogroups in the diphtheria toxoid conjugate vaccine.

\section{Guillain-Barré Syndrome (GBS) among MCV4 recipients}

In October 2005, reports suggested a possible association of GBS with MCV4, and the ACIP recommended that persons with a past history of GBS not be vaccinated with MCV4 unless they are at high risk for meningococcal disease (MMWR 2006). As of October 2007, there were 24 confirmed reports of GBS after administration of MCV4 to the US Vaccine Adverse Event Reporting System (Clark 2007). However, no cases of GBS were reported among 11- to 19-year-olds within 6 weeks after more then 200,000 doses of MCV4 from the Vaccine Safety Datalink project (Clark 2007). The excess risk of GBS is currently estimated to be from 0.4 to 1.3 per million doses (Clark 2007). The benefits of MCV4 are currently considered to significantly outweigh the potential risks, except in patients with a prior history of GBS (see contraindications and precautions).

\section{Indications for use of MCV4}

Table 2 lists the recommendations for the use of MCV4 made by the CDC Advisory Committee on Immunization Practices. It is routinely recommended for all US children aged 11-18 years, including those at additional risk. The vaccine is now recommended for use in at-risk children ages 2-10 years (MMWR 2007) and at-risk adults aged 19-55 years. Persons aged 2-55 years who continue to be at risk for meningococcal
Table 2 Recommendations for use of tetravalent (A, C,Y,W-I35) meningococcal conjugate vaccine

\begin{tabular}{|c|c|}
\hline Population type & Ages (years) \\
\hline \multicolumn{2}{|l|}{ Routine vaccination } \\
\hline All persons aged II-18 years & $11-18$ \\
\hline \multicolumn{2}{|l|}{ Persons at increased risk for meningococcal disease } \\
\hline College freshmen living in dormitories & $19-55$ \\
\hline Military recruits & $19-55$ \\
\hline $\begin{array}{l}\text { Microbiologists routinely exposed to isolates } \\
\text { of Neisseria meningitidis }\end{array}$ & $19-55$ \\
\hline $\begin{array}{l}\text { Persons with anatomic or functional asplenia or } \\
\text { terminal complement deficiencies }\end{array}$ & $2-55$ \\
\hline $\begin{array}{l}\text { Travelers to or residents of countries in } \\
\text { which N. meningitidis is hyperendemic or epidemic, } \\
\text { especially sub-Saharan Africa } \\
\text { during the dry season (December-June) or } \\
\text { Saudi Arabia during the Hajj }\end{array}$ & $2-55$ \\
\hline Persons infected with $\mathrm{HIV}^{\mathrm{a}}$ & $2-55$ \\
\hline
\end{tabular}

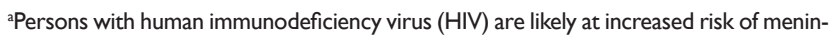
gococcal disease, although not to the extent that they are at risk for invasive Streptococcus pneumoniae infection.The efficacy of MCV4 among HIV-infected persons is unknown. Derived from Bilukha and Rosenstein (2005) with update from MMWR (2007).

infection, despite having been vaccinated with MPSV4 3 or more years earlier, are also candidates for revaccination with MCV4 (Bilukha and Rosenstein 2005). The FDA has not approved the vaccine for use in children younger then 2 years or adults older then 55 years. Consequently MPSV4 could be substituted for adults older than 55 years.

\section{Contraindications and precautions to use of MCV4}

Administration of MCV4 is contraindicated among persons known to have a severe allergic reaction to any component of the vaccine, including diphtheria toxoid or to natural latex rubber. MCV4 is an inactivated vaccine and may be given to immunosuppressed persons, but this may result in suboptimal immune response. No data are available about its safety during pregnancy (Bilukha and Rosenstein 2005). A history of GBS is considered a precaution to administering MCV4, and MPSV4 is an acceptable short-term alternative providing protection for approximately 3-5 years (MMWR 2007).

\section{Cost effectiveness of MCV4}

Cost effectiveness of meningococcal vaccination has been somewhat controversial, especially with the polysaccharide MPSV4 vaccine in developed countries where the vast majority of meningococcal cases are sporadic. One analysis estimated that vaccinating all US college freshmen living in dormitories with MPSV4 would cost US\$0.6-\$1.9 million per case averted and US\$7-20 million per death averted 
(Scott 2002). This analysis did not consider the cost of chemoprophylaxis or vaccination in the event of an outbreak, the cost of rehabilitating IMD survivors with sequelae, and the anxiety and stress due to an outbreak in the university community (Scott 2002). It has been estimated that routine vaccination of US adolescents 11 years old with MCV4 would prevent 270 cases of meningococcal disease and 36 deaths over 22 years at a cost of US\$633,000 per case and US\$121,000 per life-year saved (Shepard et al 2005). Although financial costs of prevention are high, the mortality and morbidity of IMD warrant the routine use of MCV4 in adolescents.

\section{Conclusion and future issues}

In addition to the devastation of the disease, considerable anxiety often accompanies an outbreak of IMD in the community or affected regions. The MCV4 has demonstrated immunogenicity to serogroups A, C, Y, and W-135 with an acceptable degree of reactogenicity. Based on the experience with conjugated meningococcal $\mathrm{C}$ vaccine in the UK, it is believed that MCV4 will have a similar impact on IMD caused by those serogroups. Because it is a conjugate vaccine, it is believed that the immunity provided by this vaccine will last longer then that provided by MPSV4. However, data suggest that serologic correlates of immunity to serogroup $\mathrm{C}$ may wane rapidly after 1 year in young children (Snape et al 2005; Borrow and Miller 2006). The conjugate vaccines prime for immune memory but it is not clear that this occurs quickly or sufficiently enough to provide protection from disease (Borrow and Miller 2006). Protection from disease may therefore depend on persistence of protective antibody levels and herd immunity. Thus far, antibody persistence in older children and adolescents appears good but it remains to be seen whether a booster dose of vaccine will be required at some future date.

\section{Disclosures}

Dr. Kimmel owns shares in GSK, and is a member of GSK Family/General Practitioner Advisory Board for Cervical Cancer effective February 2008. He is a consultant for Merck, Inc., and on the speaker's bureau, and a consultant for Sanofi Pasteur, and on the speaker's bureau. He is also a consultant, CDC grant to Society of Teachers of Family Medicine Foundation for project "Increasing Immunizations by Family Physicians". Richard Zimmerman, MD, MPH, University of Pittsburgh, is the primary investigator. Grant is now expired.

\section{References}

Abramson JS, Spika JS. 1985. Persistence of Neisseria meningitidis in the upper respiratory tract after intravenous antibiotic therapy for systemic meningococcal disease. J Infect Dis, 151:370-1.

Barquet N, Domingo P, et al. 1999. Meningococcal disease in a large urban population (Barcelona, 1987-1992): predictors of dismal prognosis. Barcelona Meningococcal Disease Surveillance Group. Arch Intern Med, 159:2329-40.

Bilukha OO, Rosenstein N. 2005. Prevention and control of meningococcal disease. Recommendations of the Advisory Committee on Immunization Practices (ACIP). MMWR Recomm Rep, 54(RR-7):1-21.

Borrow R, Andrews N, et al. 2001. Serological basis for use of meningococcal serogroup $\mathrm{C}$ conjugate vaccines in the United Kingdom: reevaluation of correlates of protection. Infect Immun, 69:1568-73.

Borrow R, Balmer P, et al. 2005. Meningococcal surrogates of protection serum bactericidal antibody activity. Vaccine, 23:2222-7.

Borrow R, Miller E. 2006. Long-term protection in children with meningococcal C conjugate vaccination: lessons learned. Expert Rev Vaccines, 5:851-7.

Bruce MG, Rosenstein NE, et al. 2001. Risk factors for meningococcal disease in college students. JAMA, 286:688-93.

Campbell JD, Edelman R, et al. 2002. Safety, reactogenicity, and immunogenicity of a tetravalent meningococcal polysaccharidediphtheria toxoid conjugate vaccine given to healthy adults. $J$ Infect Dis, 186:1848-51.

[CDC] Centers for Disease Control and Prevention. 2007. Active Bacterial Core Surveillance Report, Emerging Infections Program Network, Neisseria meningitidis, 2006 [online]. Accessed 26 Mar 2008. URL: http://www.cdc.gov/ncidod/dbmd/abcs/survreports/ mening06.pdf.

Clark T. 2007. Meningococcal conjugate vaccine and Guillain Barre syndrome. Advisory Committee on Immunization Practices meeting Oct 24 2007, Atlanta, Georgia, USA.

Condon RJ, Riley TV, et al. 1994. Invasive meningococcal infection after splenectomy. BMJ, 308:792-3.

Danzig L. 2004. Meningococcal vaccines. Pediatr Infect Dis J, 23(12 Suppl):S285-92.

El Bashir H, Heath PT, et al. 2006. Antibody responses to meningococcal (groups A, C, Y and W135) polysaccharide diphtheria toxoid conjugate vaccine in children who previously received meningococcal $\mathrm{C}$ conjugate vaccine. Vaccine, 24:2544-9.

EU-IBIS. 2006. Invasive Neisseria meningitidis in Europe - 2006 [online]. Accessed Apr 5 2008. URL: www.euibis.org.

Gardner P. 2006. Clinical practice. Prevention of meningococcal disease. N Engl J Med, 355:1466-73.

Girgis N, Frenck RW Jr, et al. 1998. Azithromycin compared with rifampin for eradication of nasopharyngeal colonization by Neisseria meningitidis. Pediatr Infect Dis J, 17:816-9.

Goldschneider I, Gotschlich EC, et al. 1969a. Human immunity to the meningococcus. I. The role of humoral antibodies. J Exp Med, 129:1307-26.

Goldschneider I, Gotschlich EC, et al. 1969b. Human immunity to the meningococcus. II. Development of natural immunity. J Exp Med, 129:1327-48.

Gotschlich EC, Goldschneider I, et al. 1969. Human immunity to the meningococcus. IV. Immunogenicity of group A and group C meningococcal polysaccharides in human volunteers. J Exp Med, 129:1367-84.

Granoff DM. 2004. Meningococcal vaccines. In Plotkin SA, Orenstein W, Offit PA (eds).Vaccines, 4th ed. Philadelphia, PA: Elsevier-Saunders: 959-87.

Granoff DM, Gupta RK, et al. 1998. Induction of immunologic refractoriness in adults by meningococcal C polysaccharide vaccination. J Infect Dis, 178:870-4.

Granoff DM, Pollard AJ. 2007. Reconsideration of the use of meningococcal polysaccharide vaccine. Pediatr Infect Dis J, 26:716-22. 
Hahne S, Gray SJ, et al. 2002. W135 meningococcal disease in England and Wales associated with Hajj 2000 and 2001. Lancet, 359:582-83.

Harrison LH, Dwyer DM, et al. 1999. Risk of meningococcal infection in college students. JAMA, 281:1906-10.

Harrison LH, Pass MA, et al. 2001. Invasive meningococcal disease in adolescents and young adults. JAMA, 286:694-9.

Jodar L, Feavers IM, et al. 2002. Development of vaccines against meningococcal disease. Lancet, 359:1499-508.

Keyserling H, Papa T, et al. 2005. Safety, immunogenicity, and immune memory of a novel meningococcal (groups A, C, Y, and W-135) polysaccharide diphtheria toxoid conjugate vaccine (MCV-4) in healthy adolescents. Arch Pediatr Adolesc Med, 159:907-13.

MacDonald NE, Halperin SA, et al. 1998. Induction of immunologic memory by conjugated vs plain meningococcal $\mathrm{C}$ polysaccharide vaccine in toddlers: a randomized controlled trial. JAMA, 280:1685-9.

Maiden MC, Stuart JM. 2002. Carriage of serogroup C meningococci 1 year after meningococcal $\mathrm{C}$ conjugate polysaccharide vaccination. Lancet, 359:1829-31.

MMWR. 2000. Prevention and control of meningococcal disease. Recommendations of the Advisory Committee on Immunization Practices (ACIP). MMWR Morb Mortal Wkly Rep, 49(RR07):1-10.

MMWR. 2001a. Exposure to patients with meningococcal disease on aircrafts - United States, 1999-2001. MMWR Morb Mortal Wkly Rep, 50:485-9.

MMWR. 2001b. Public health dispatch: update: assessment of risk for meningococcal disease associated with the Hajj 2001. MMWR Morb Mortal Wkly Rep, 50:221-2.

MMWR. 2006. Update: Guillain-Barre syndrome among recipients of Menactra meningococcal conjugate vaccine - United States, June 2005-September 2006. MMWR Morb Mortal Wkly Rep, 55:1120-4.

MMWR. 2007. Notice to readers: recommendation from the advisory committee on immunization practices (ACIP) for use of quadrivalent meningococcal conjugate vaccine (MCV4) in children aged 2-10 years at increased risk for invasive meningococcal disease. MMWR Morb Mortal Wkly Rep, 56:1265-6.

MMWR. 2008. Emergence of fluoroquinolone-resistant Neisseria meningitidis - Minnesota and North Dakota, 2007-2008. MMWR Morb Mortal Wkly Rep, 57:173-5.

Neal KR, Nguyen-Van-Tam JS, et al. 2000. Changing carriage rate of Neisseria meningitidis among university students during the first week of term: cross sectional study. BMJ, 320:846-9.

O'Hallahan J, Lennon D, et al. 2004. The strategy to control New Zealand's epidemic of group B meningococcal disease. Pediatr Infect Dis J, 23(12 Suppl):S293-8.

Pichichero M, Casey J, et al. 2005. Comparative trial of the safety and immunogenicity of quadrivalent (A, C, Y, W-135) meningococcal polysaccharidediphtheria conjugate vaccine versus quadrivalent polysaccharide vaccine in two- to ten-year-old children. Pediatr Infect Dis J, 24:57-62.
Pichichero M, Papa T, et al. 2006. Immune memory in children previously vaccinated with an experimental quadrivalent meningococcal polysaccharide diphtheria toxoid conjugate vaccine. Pediatr Infect Dis J, 25:995-1000.

Pollard AJ. 2004. Global epidemiology of meningococcal disease and vaccine efficacy. Pediatr Infect Dis J, 23(12 Suppl):S274-9.

Pollard AJ, Frasch C. 2001. Development of natural immunity to Neisseria meningitidis. Vaccine, 19:1327-46.

Ramsay ME, Andrews N, et al. 2001. Efficacy of meningococcal serogroup $\mathrm{C}$ conjugate vaccine in teenagers and toddlers in England. Lancet, 357:195-6.

Ramsay ME, Andrews NJ, et al. 2003. Herd immunity from meningococcal serogroup C conjugate vaccination in England: database analysis. BMJ, 326:365-6.

Rennels M, King J Jr, et al. 2004. Dosage escalation, safety and immunogenicity study of four dosages of a tetravalent meninogococcal polysaccharide diphtheria toxoid conjugate vaccine in infants. Pediatr Infect Dis $J, 23: 429-35$.

Rosenstein N, Levine O, et al. 1998. Efficacy of meningococcal vaccine and barriers to vaccination. JAMA, 279:435-9.

Rosenstein NE, Perkins BA, et al. 2001. Meningococcal disease. N Engl J Med, 344:1378-88.

Scott RD, Meltzer MJ, et al. 2002. Vaccinating first-year college students living in dormitories for meningococcal disease. Am J Prev Med, 23:98-105.

Shepard CW, Ortega-Sanchez IR, et al. 2005. Cost-effectiveness of conjugate meningococcal vaccination strategies in the United States. Pediatrics, 115:1220-32.

Snape MD, Kelly DF, et al. 2005. Lack of serum bactericidal activity in preschool children two years after a single dose of serogroup C meningococcal polysaccharide-protein conjugate vaccine. Pediatr Infect Dis J, 24:128-31.

Trotter CL, Andrews NJ, et al. 2004. Effectiveness of meningococcal serogroup $\mathrm{C}$ conjugate vaccine 4 years after introduction. Lancet, 364:365-7.

van Deuren M, Brandtzaeg P, et al. 2000. Update on meningococcal disease with emphasis on pathogenesis and clinical management. Clin Microbiol Rev, 13:144-66.

Yazdankhah SP, Kriz P, et al. 2004. Distribution of serogroups and genotypes among disease-associated and carried isolates of Neisseria meningitidis from the Czech Republic, Greece, and Norway. J Clin Microbiol, 42:5146-53. 
